\title{
In-situ observation of the in-plane field induced nucleation of skyrmion using Lorentz-TEM
}

Binbin wang ${ }^{1}$, Po-kuan $\mathrm{Wu}^{1}$, Núria Bagués ${ }^{2}$, Qiang Zheng ${ }^{3}$, Jiaqiang Yan ${ }^{3}$, Mohit Randeria ${ }^{4}$ and David $\mathrm{McComb}^{2}$

${ }^{1}$ The Ohio State University, COLUMBUS, Ohio, United States, ${ }^{2}$ Center for Electron Microscopy and Analysis, The Ohio State University, Columbus, OH, USA, COLUMBUS, Ohio, United States, ${ }^{3}$ Oak Ridge National Laboratory, United States, ${ }^{4}$ The Ohio State University, United States

Exploiting magnetism for low-power spin-based devices is a topic of growing interest, and magnetic skyrmions have been actively investigated for their unique properties, which are topologically protected non-volatile vortex-like spin textures [1]. To realize the potential of skyrmion-based devices it is essential to identify materials where small, stable skyrmions can be both created and manipulated in a reliable way. Lorentz transmission electron microscopy (LTEM) can be used to investigate the dynamics of skyrmion nucleation and manipulation in such materials in real space, an important step towards the development of novel spintronic devices. [2,3]

In this work, we have employed LTEM to probe transformations of the magnetic texture in a centrosymmetric magnet, $\mathrm{Fe}_{3} \mathrm{Sn}_{2}$, with a sample that has an engineered thickness gradient. $\mathrm{Fe}_{3} \mathrm{Sn}_{2}$ has a layered rhombohedral crystal structure comprising kagome FeSn bilayers sandwiched between honeycomb Sn layers along the caxis. $\mathrm{Fe}_{3} \mathrm{Sn}_{2}$ is a noncollinear frustrated ferromagnet with a Curie temperature of $640 \mathrm{~K}$. It undergoes a spin reorientation that transforms from the magnetically easy ab-plane to the c-axis as the temperature increases [4]. These transformations give rise to a wealth of intrinsic magnetic configurations in a wide temperature range, including a skyrmion phase stable of a wide stable temperature range, $130 \mathrm{~K}-630 \mathrm{~K}$ [5]. However, in the absence of geometric confinement, the critical field for nucleation of isolated skyrmions in this system is rather high $(\sim 800 \mathrm{mT})$. In this contribution, we report that dynamic switching of a small in-plane magnetic field $(\sim 5 \mathrm{mT})$ can induce a reversible magnetic phase transformation at the interface between differently oriented magnetic domains, as shown in Fig.1.

Single crystals of $\mathrm{Fe}_{3} \mathrm{Sn}_{2}$ were grown out of $\mathrm{Sn}$ flux using liquid transport growth technique and thin lamella specimens were prepared using a focused ion beam (FIB). The engineered thickness gradient was created during the final thinning process in FIB and measured using electron energy-loss spectroscopy. To observe the in-situ dynamic behavior of magnetic spin textures under the influence of in-plane magnetic field, LTEM images were recorded as the in-plane field was oscillated with a small fixed normal field. The in-situ processes were recorded using a Gatan K2 direct electron detector attached to a ThermoFisher Titan aberration-corrected TEM. As shown in Fig.2, micromagnetic simulations and associated experiments reveal that the thickness dependence of the response to the external in-plane field is the main factor for the skyrmion formation. It is also found that magnetic texture transformation is related to the observed reorientation of magnetic stripes, which suggests materials with rotatable anisotropy are promising candidates as skyrmion materials. This research establishes that magnetic skyrmion nucleation and manipulation can be controlled using in-plane fields, providing great promise as a new approach to design spintronic devices.

The authors acknowledge funding from DARPA under Grant No. D18AP00008; The Center for EmergentMaterials at The Ohio State University, an NSF MRSEC (DMR-2011876); Work at ORNL was supported by theU.S. Department of Energy, Office of Science, Basic Energy Sciences, Materials Sciences and Engineering Division. 

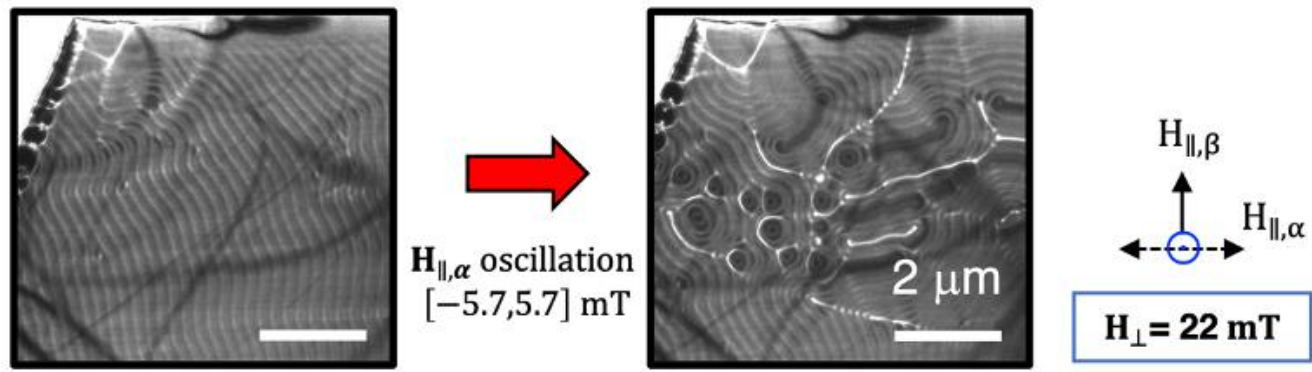

Figure 1. Observed magnetic texture transformation at magnetic domain boundaries during the in-plane field oscillation process. The external magnetic field was fixed at $22 \mathrm{mT}$ while the in-plane field oscillation was performed along $\mathrm{H} \|, \alpha$ directions by sample tilting.

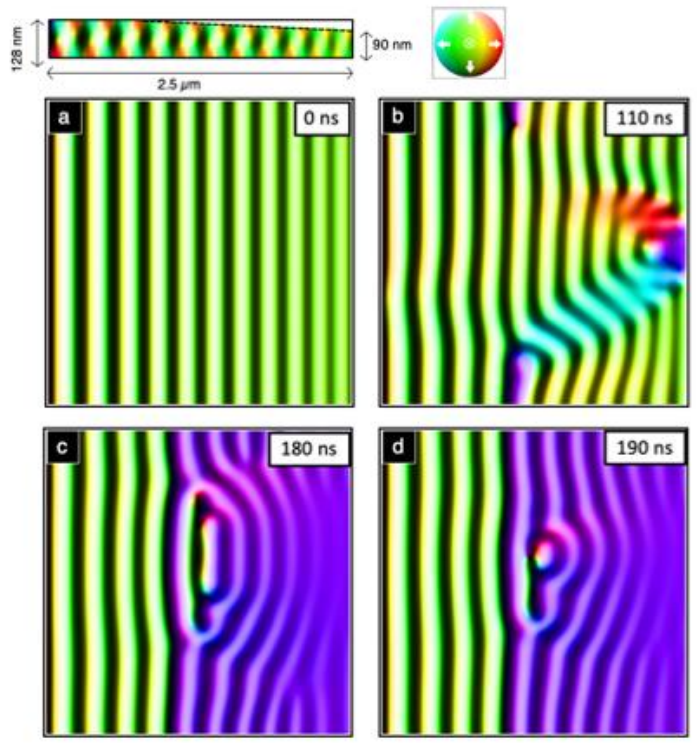

Figure 2. Snapshots of dynamic transformation between magnetic bubbles and stripes in micromagnetic simulation while the external field changes from $2 \mathrm{mT}$ to $-10 \mathrm{mT}$ in y direction in $190 \mathrm{~ns}$. The color in images indicates spin directions as shown in color wheel, while the darkness/white indicates out-of-plane magnetizations. a, Initial stripe at $0 \mathrm{~ns}$ paralleled states after annealing from a strong field to $2 \mathrm{mT}$ and equilibrated. The initial configuration is prepared with field annealing from strong in-plane field along direction. The inset above, which is the cut view, shows the thickness gradient (from $128 \mathrm{~nm}$ to $90 \mathrm{~nm}$ ) for simulation setup. b-d, The snapshots at $110 \mathrm{~ns}, 180 \mathrm{~ns}$, and $190 \mathrm{~ns}$. Shown in b one can see that the in-plane component inversion starts from the thinner end. The inversion of the in-plane component and distortion of stripes result in skyrmions as shown in c and d. In c, two clear domains and half target like skyrmion structure are forming near the boundary and it shrinks into a skyrmion as time goes.

\section{References}

[1] N. Nagaosa, Y. Tokura, Nat. Nanotechnol. 8 (2013), p.899-911.

[2] X.Z. Yu, et al., Nature. 465 (2010), p. 901-904.

[3] L. Peng, et al., Nano Lett. 18 (2018), p. 7777-7783.

[4] L.A. Fenner, et al., J. Phys.: Condens. Matter. 21 (2009), p. 452202.

[5] Z. Hou, et al., Adv. Mater. 29 (2017), p.1701144. 